Cultures \& Conflits

53 | printemps 2004

Surveillance politique : regards croisés

\title{
Figures de l'officier traitant à travers les archives de la Stasi
}

Sonia Combe

\section{OpenEdition \\ 12 Journals}

Édition électronique

URL : http://journals.openedition.org/conflits/999

DOI : $10.4000 /$ conflits.999

ISSN : $1777-5345$

Éditeur :

CCLS - Centre d'études sur les conflits lilberté et sécurité, L'Harmattan

Édition imprimée

Date de publication : 1 mars 2004

Pagination : 99-112

ISBN : 2-7475-6791-5

ISSN : 1157-996X

Référence électronique

Sonia Combe, «Figures de l'officier traitant à travers les archives de la Stasi », Cultures \& Conflits [En ligne], 53 I printemps 2004, mis en ligne le 04 octobre 2004, consulté le 30 mars 2021. URL : http:// journals.openedition.org/conflits/999; DOI : https://doi.org/10.4000/conflits.999

Ce document a été généré automatiquement le 30 mars 2021.

Creative Commons License 


\title{
Figures de l'officier traitant à travers les archives de la Stasi
}

\author{
Sonia Combe
}

1 L'ouverture immédiate, au lendemain même de la chute du mur de Berlin, des dossiers de la police politique est-allemande a constitué pour la recherche sur la surveillance politique et policière une chance unique et inespérée. Une abondante littérature savante atteste que les chercheurs ont su s'en saisir ${ }^{1}$. L'exemple restant sans précédent (les autres exemples de « démocraties populaires » n'ont pas encore donné semblable accès à leurs archives), il est trop tôt pour introduire une dimension comparatiste et, faute de sources de même nature, on s'abstiendra de généralisations hâtives fondées sur des idées préconçues. Tout en sachant que la Segurimi albanaise ou la Securitate roumaine, ou toute autre police politique dans les Etats de type soviétique occupait la même fonction que la Stasi, la question du degré de participation de chaque société à la surveillance reste ouverte. Toutefois, dans le cas de l'Allemagne de l'Est, une comparaison historique était inévitable. On doit à Robert Gellately un constat précieux pour la compréhension du fonctionnement interne de la RDA : selon ce spécialiste du Troisième Reich, contrairement à ce qui se passa sous le régime nazi où la délation fut un phénomène social spontané, en Allemagne de l'Est, il fallut l'organiser'2 ${ }^{2}$ Ce fut là l'oeuvre des "officiers traitants" dont l'écrivain tchèque (et piètre informateur), Bohumil Hrabal, nous a sans doute donné à lire l'un des rares portraits dans cette nouvelle « confessante » empreinte d'auto-ironie qu'il eut le courage d'écrire peu avant sa mort ${ }^{3}$. Car on s'interroge généralement peu sur ce personnage chargé d'organiser la surveillance et la délation, personnage de l'ombre par excellence qui entretient des contacts avec des informateurs ou mouchards, qui rédige des rapports tamponnés d'un «strictement secret » et décide le cas échéant d'un châtiment. Cet homme (dans la plupart des cas il s'agit d'un homme, le «renseignement » faisant partie des tâches essentiellement masculines) ne s'est guère livré depuis la chute d'un régime qu'il a le plus souvent servi sans état d'âme - si ce n'est en son âme et conscience. En dépit de l'aspect sulfureux du sujet, les médias ne sont pas parvenus à " arracher " plus d'une demi-douzaine de témoignages d'anciens officiers lesquels, pourtant, avaient atteint l'effectif de 80000 à l'automne 1989. Dans leur majorité, ces hommes de peine de la 
surveillance politique sont apparus comme des personnalités ordinaires, plus naïfs que machiavéliques, mais d'une naïveté qui n'avait d'égale que leur loyauté à l'égard du régime, et aucun d'eux, naturellement, ne confessa d'horribles crimes $^{4}$. On peut supposer qu'il leur était difficile de porter un jugement a posteriori sur une activité qu'ils avaient considérée comme normale et légitime aussi longtemps qu'ils l'avaient exercée. Quant à leurs chefs, on n'en pût rien tirer qui nous aurait davantage éclairé pour comprendre le fonctionnement interne d'une institution-type des pays soumis à l'ordre soviétique. Le patron de la Stasi, dont les activités s'exerçaient à l'intérieur du pays, Erich Mielke, se réfugia dans le gâtisme, vrai ou simulé mais crédible, non seulement du fait de son âge avancé et de son emprisonnement, mais du fait du choc psychique lié à l'écroulement de la RDA. De son côté, Markus Wolf, le chef du service du contre-espionnage dont les activités, elles, avaient l'Etranger pour champ de manoeuvre, fit preuve d'une telle habileté, d'un tel humour à l'occasion, bref d'une telle intelligence, qu'il finit par susciter l'admiration de ses pires adversaires. Les archives de la Stasi restent donc le document par excellence et par défaut pour cerner la figure du Führungsoffizier - terme que nous traduisons par officier traitant, emprunt fait aux romans d'espionnage. Et bien que ces traces là de son activité ne nous permettent pas d'entrer dans son intimité, il reste possible, à travers la perception qu'il livre dans ses rapports écrits de ceux qui font l'objet de sa surveillance, d'établir une sorte de portrait-robot de l'officier traitant ou, du moins, d'en dégager les différentes figures rencontrées.

2 Procéder à ce renversement de regard nécessite donc une autre lecture des dossiers étudiés. Cela engage de lire ce qui est dit de la personne surveillée en réfléchissant sur ce que cela trahit non pas d'elle ou des modalités de la surveillance, mais de l'univers mental de celui qui a rédigé les rapports et comptes-rendus de rencontres - tout en gardant naturellement présent à l'esprit que cet univers mental a été modelé par et pour les nécessités de la surveillance - ainsi que de sa contribution au maintien d'une société réprimée et répressive. M'étant appuyée sur une vingtaine de dossiers de personnalités du monde universitaire et académique qui avaient eu des responsabilités d'encadrement ${ }^{5}$, il est possible qu'au cours de mes recherches je n'ai pas rencontré l'officier traitant "moyen ", mais des hommes que leur hiérarchie avait estimé aptes à nouer des contacts avec l'élite intellectuelle du pays. Dans deux cas, j'ai même eu la certitude que les officiers traitants avaient fait des études avec les personnes qu'ils surveillaient, l'un en philosophie, l'autre en histoire. Sans donc pouvoir faire la sociologie de cette catégorie sociale ${ }^{6}$, en rappelant le contexte dans lequel se constitua et évolua ce corps très spécial de fonctionnaires, une représentation schématique reste possible.

Fondation et formation

3 Créée en 1950 sur le modèle de la Tchéka, la Stasi (abréviation de Ministerium für Staatssicherheit, ministère pour la Sécurité de l'Etat) connaît une évolution de ses fonctions adaptées aux différentes étapes de construction de la RDA : de sa fondation à 1958 (affaires Harich et Ernst Bloch) ${ }^{7}$, elle exerce une répression brutale et ouverte contre tous les opposants - réels ou putatifs - au régime; de 1958 au début des années 1970, d'institution répressive, elle se transforme progressivement en organe de surveillance; de 1970 à 1989, elle perfectionne sa fonction de surveillance au point de devenir le panopticon de la société est-allemande grâce au quadrillage du pays par un réseau d'informateurs non officiels ${ }^{8}$ : l'état permanent de visibilité dans lequel se 
trouve la population - qui sait pouvoir faire l'objet, à tout moment, d'une surveillance de la vie sociale (plus rarement privée ${ }^{9}$ - permet ce que Foucault appela le « fonctionnement automatique du pouvoir $~{ }^{10} \mathrm{et}$ rend superflue la fonction répressive, cette dernière n'étant plus exercée que de façon ciblée et sélective. Elle est alors destinée à réactiver la mémoire collective de la terreur que la Stasi a fait régner dans les années de consolidation du régime (années 1950). En quarante ans, la surveillance politique est devenue une fin en soi, et la Stasi essentiellement une police d'observation. Pour preuve, le 4 novembre 1989, lors de la manifestation sur l'Alexanderplatz, prélude à la chute du Mur cinq jours plus tard, la Stasi est au courant de tout mais incapable d'entreprendre quoi que ce soit. Les quatre millions de dossiers qu'elle a fabriqués ne lui ont été d'aucune utilité, attestant par là même l'inanité d'une surveillance politique et policière aussi raffinée.

En quarante ans, l'officier de la Stasi a, lui aussi, évolué. Il ne pouvait se contenter d'être seulement brutal. Dès lors que la violence devait perdre de sa visibilité et que les sanctions « douces » (mises à l'écart, mises au placard, harcèlement administratif, etc.) s'étaient substituées à la répression classique, il lui fallait perfectionner ses méthodes, devenir plus discret et plus efficace à la fois. En 1951 avait été créée, à Potsdam-Eiche, la "juristische Hochschule ", école supérieure de droit. Sous ce nom anodin se cachait l'école des cadres de la Stasi. En 1965, cette dernière deviendra ni plus ni moins et tout aussi officiellement « établissement universitaire » et, en 1968, on introduira des cours de psychologie (dite "marxiste-léniniste») pour apprendre "la psyché de l'ennemi ». L'enseignement devient pluridisciplinaire, on y enseigne la sociologie et la communication de masse, les techniques d'influence, on y apprend à évaluer les personnalités (pratique de tests), à conduire des entretiens, et aussi ces fameuses Zersetzungsmassnahmen, ou mesures visant à déstabiliser/détruire la personnalité. Au nombre de ces mesures, les rumeurs que l'on fait courir sur le dissident selon lesquelles, par exemple, il serait un informateur de la Stasi, provoquant ainsi sans explication son isolement et sa mise à l'écart par son entourage ; ou encore, sur des pratiques sexuelles particulières; ou enfin, dans le milieu universitaire, la rumeur, très efficace, qui se chuchote de bouche à oreilles et se répand avec une facilité inquiétante, concernant une "baisse de niveau » de l'intellectuel dont les idées critiques seraient parvenues à la Stasi, ou bien qui aurait été dénoncé comme esprit critique par l'un de ses collègues pour de simples raisons de rivalité. D'autres mesures pouvaient être plus raffinées encore, celles du cambriolage qui ne laisse pas de traces: un premier cambriolage sans effraction, portait, par exemple, sur des objets intimes comme les gants de toilette; un deuxième, trois mois plus tard, sur les torchons à imprimé écossais ; un troisième, sur des taies d'oreiller. Un quatrième cambriolage aurait été, selon la Stasi, inutile, la personnalité des occupants de l'appartement cambriolé ayant été atteinte au point de les rendre totalement inoffensifs : ne sachant d'où viennent les coups, passant pour fou auprès de son entourage, le couple ainsi visé dans la part la plus intime de son intimité, l'intimité corporelle, serait bel et bien devenu paranoïaque, but de la Stasi. Variante "douce " de la psychiatrisation des dissidents en URSS, les Zersetzungsmassnahmen correspondent à la forme la plus achevée de l'oeuvre criminelle de la Stasi.

L'officier traitant et son double

5 On distinguera les quatre figures suivantes de l'officier traitant: le biographe, le conformiste, le confident, l'instance morale. Si tous les officiers traitants partagent la fonction de biographe, à l'inverse, tous ne partagent pas celle d'instance morale (bien 
qu'une fonction correctrice soit décelable dans plus d'une relation entre l'officier et son double). Cette figure inattendue mérite cependant d'être soulignée car elle ne semble pas relever de l'exception. Mais tout d'abord, comment s'élabore un dossier de surveillance? Un officier de la Stasi est chargé de recruter un informateur. Si la personne contactée accepte la collaboration, elle aura pour tâche de produire, à intervalles plus ou moins réguliers, un rapport sur d'autres personnes. Dans le cas des universitaires, il était rare qu'ils prennent la peine d'écrire. L'officier rencontrait son informateur et faisait ensuite un compte-rendu de leur rencontre. Il ajoutait au dossier les rapports ou documents éventuellement rapportés par l'informateur et faisait enfin un autre rapport sur ce dernier. Il faut en effet se souvenir qu'un informateur était, lui aussi, toujours objet de surveillance (conformément à l'adage «Faire confiance, c'est bien, contrôler, c'est mieux »). La constitution du dossier était régulièrement ponctuée par des «bilans intermédiaires » que l'officier rédigeait pour faire le point, mettre un terme à une collaboration, une surveillance ou, au contraire, l'intensifier. Dans de nombreux cas, on discerne la trace d'une lecture "supervisante ", sous la forme du coup de crayon rouge du supérieur hiérarchique qui contrôle le travail de son subordonné. Cette surveillance à tous les niveaux accroissait le travail de la Stasi, mais avait pour but de faciliter la transmission des dossiers: pour des surveillances de longue durée, officiers et informateurs se succédaient.

\section{Le biographe}

7 Bien des dossiers se présentent comme des manuscrits pratiquement prêts pour l'édition. Sur la page de garde figurent toutes les informations nécessaires: nom de l'auteur (ou des auteurs lorsque la surveillance a mobilisé plusieurs officiers), titre (sous la forme du nom ou pseudonyme de la personne surveillée), index des noms cités, pagination, et même les sources ${ }^{11}$. Nous nous trouvons ainsi face à la biographie d'une personne vivante, rédigée à son insu par une plume policière. Cette dernière présente les mêmes caractéristiques que toutes les autres plumes policières : elle démontre une collecte compulsive d'informations qui trahit un fétichisme du détail. Pour comparaison, les archives du FBI sur la période de la chasse aux sorcières menée par le sénateur Mc Carthy dans les années 1950 en témoignent pareillement ${ }^{12}$. Mais c'est surtout la pauvreté de la langue qui est la principale caractéristique des "récits de vie " rédigés par la Stasi. Une pauvreté qui renvoie à celle de l'univers mental de ses officiers, au caractère primitif de leur système de représentation lequel se compose $\mathrm{d}^{\prime}$ '« amis » et d'« ennemis » au comportement « positif » ou « négatif ». Construit sur le mode d'opposition binaire, le vocabulaire de l'officier de la Stasi se structure autour de termes associés comme: amis/ennemis; positif/négatif; humaniste/ individualiste ; révisionniste/subjectiviste; progressiste (c'est-à-dire socialiste)/ esprit critique, etc. Enfin, un adjectif revient souvent, celui de « dépravé » caractérisant l'« ennemi ». Il y aurait beaucoup à dire sur l'affection pour ce mot de la part d'hommes dont les méthodes de travail misaient et exploitaient précisément l'amoralisme...

8 A la pauvreté de vocabulaire s'ajoute de façon prévisible la faiblesse de la culture générale. Dans un rapport, Kafka et Proust (orthographié "Brust») se trouvent désignés comme des auteurs ouest-allemands contemporains ; dans un autre, à côté du nom de Walter Benjamin (cité par la personne qui fait l'objet de la surveillance), l'officier traitant a écrit, soulignant le nom au crayon rouge : «à surveiller ». La plupart des noms de lieux étrangers le plus souvent retranscris de façon phonétique comportent, de façon presque systématique, des fautes d'orthographe. Mais surtout, le 
«roman » qu'écrit l'officier de la Stasi est un roman policier : il s'agit de dessiner la figure d'un ennemi en construisant son acte d'accusation. Le policier, il convient de le rappeler, n'est pas à la recherche de la vérité mais à la recherche d'un coupable (ce qui n'exclue pas les nombreux cas de dossiers fermés «faute de preuves " sans plus d'explications, bien que tout laisse entendre que c'est à l'intervention du Parti que le « suspect » devait cette issue). D'où l'aspect doublement fictionnel de la «biographie » ainsi rédigée.

Le conformiste

Dans le «profil psychologique » que l'officier traitant dresse de l'informateur ou de la personne surveillée, on retrouve les mêmes critères d'évaluation: l'aspect extérieur (tenue vestimentaire et coupe de cheveux), la mise de drapeaux (drapeau rouge et drapeau de la RDA) à la fenêtre du domicile privé les jours de fête, le comportement avec les voisins et les subordonnés à l'université ou à l'Académie des sciences ${ }^{13}$, le rapport aux biens matériels, essentiellement la voiture. Regard conformiste et petitbourgeois, ce regard social est, au départ, plus proche de celui de l'homme de la rue que d'une police politique. Si l'examen du train de vie peut fournir des informations sur l'éventuel degré de corruptibilité de la personne surveillée, la voiture, elle, souvent mentionnée comme objet-culte de l'intellectuel, est dans le collimateur de l'officier car elle suscite l'envie, les universitaires ayant certainement eu plus de facilités pour s'en offrir une que le simple officier de la Stasi. Ce dernier traque aussi les signes d'arrogance, celle de ceux qui exhibent leurs titres scolaires dont la Stasi, au demeurant, reste respectueuse. La personne surveillée est appelée "Doctor» (Dr) ou «Professor Dr » aussi longtemps qu'aucun acte d'accusation n'est retenu contre elle. Le fait qu'elle perde son titre au cours de la rédaction de son dossier est l'indice le plus sûr que la Stasi s'oriente vers l'inculpation. Quant à la sortie des drapeaux les jours de fête, l'officier en tient compte comme d'un signe extérieur de loyauté vis-à-vis du régime sans doute rassurant, mais pas forcément convaincant.

11 Conformément à la personnalité du petit-bourgeois conformiste, dans son jugement sur les femmes, l'officier de la Stasi apparaît comme un « chauviniste-mâle » traditionnel. Grattez le communiste, aurait dit Lénine et vous trouverez le philistin: dans son attitude vis à vis des femmes. D'une sociologue travaillant sur la place des femmes dans la société est-allemande, il est dit qu'elle a énoncé « une théorie dangereuse selon laquelle l'égalité des sexes ne signifierait pas forcément l'émancipation des femmes, et dans laquelle elle ne cite pas assez Engels $»^{14} \ldots$ Le reproche est explicite : on leur a donné l'égalité, voilà qu'elles nous parlent d'émancipation! Le fait que la nuance soit perceptible pour un policier montre d'ailleurs que ce débat a été vivant dans la société est-allemande où s'était développée une intéressante littérature féminine.

Bien que l'officier s'en tienne, à sa façon, à la consigne d'objectivité et que sa plume reste plutôt neutre, l'homophobie est elle aussi aisément décelable. Mais il traque l'homosexualité comme il traque à l'occasion les infidélités conjugales qui peuvent se transformer en moyen de chantage. C'est aussi son regard petit-bourgeois qui prête aux adeptes de Freud (à peine toléré) et à la psychanalyse (quasi inexistante) des vertus proches de la magie: lorsque échouent les tentatives de constitution de dossiers d'accusation contre un psychologue (qui avait en traitement des femmes de hauts fonctionnaires de l'Etat et dont la Stasi redoutait qu'il sache "tout») et, dix ans plus tard dans un autre contexte, contre une enseignante de l'université Humboldt (qui " parle beaucoup de psychanalyse à ses étudiants »), les échecs sont attribués au pouvoir de 
ces deux personnes de "lire dans les têtes ". Elles auraient même percé à jour " grâce à la psychanalyse " ("et au whisky» - boisson occidentale - dans le cas de la dernière) les informateurs que la Stasi leur avait dépêchés! Enfin, plus d'une fois, la plume policière trahit un penchant nationaliste. On peut en effet se demander si la haine que voue manifestement l'officier qui la surveille à Karola Bloch, épouse du philosophe Ernst Bloch, n'est pas ancrée dans ce propos rapporté et souligné au crayon rouge dans un rapport la concernant : « à propos de la mort de ses parents à Auschwitz, elle a dit que tous les Allemands étaient des salauds ". Quant à Hans Mayer, également d'origine juive et spécialiste de littérature à l'université de Leipzig, qui se sait surveillé et le dit ouvertement, l'officier de la Stasi payé pour savoir que cela est vrai, trouve le cynisme de le dire atteint de paranoïa : «Il se croit observé et voit des complots antisémites partout ». personne qu'il va tenter de recruter (on n'attrape pas les mouches avec du vinaigre, encore moins en milieu académique) et de le maintenir si ce dernier accepte la collaboration. Les menaces ne sont proférées qu'en dernier recours. Le rôle de l'institution policière doit tendre à s'estomper derrière un rapport de confiance. Il est possible que son ombre ait malgré tout enveloppé ces rencontres très spéciales, mais cela dépendait vraisemblablement du degré d'affinité entre l'officier et son agent. De fait, tous les entretiens commencent par des questions personnelles. Que devient la famille de l'agent, où en sont les enfants, la santé, etc. Devant tant de sollicitude, on se laisse aller à la confidence et, plus d'une fois, on trouve dans ces dossiers la preuve que la Stasi n'était pas ingrate: elle aidait au rapatriement d'un petit héritage laissé à l'Ouest par un père, une tante, faisait en sorte qu'un fils un peu rebelle ne soit pas expulsé de l'université en raison de sa fréquentation d'Andersdenkenden (« ceux qui pensent autrement ", périphrase par laquelle se désignaient les dissidents), etc. Dans un deuxième temps seulement, on arrivait au vif du sujet, c'est-à-dire à la vie professionnelle dont l'officier allait généralement être informé de la manière la plus fidèle - même s'il est aussi vrai que certains IM évitaient délibérément de parler de leurs collègues. C'est à l'officier, oreille (presque « écoute »!) " neutre » et extérieure, qu'on allait confier ses démêlés éventuels avec les collègues, les petites et grandes blessures narcissiques qui jalonnent la vie de l'universitaire et du chercheur, les rivalités, les conflits de pouvoir. «La Stasi était mon Eckermann », a pu dire un jour un écrivain qui, contrairement à Goethe, n'avait point de secrétaire particulier. Plus d'une personne a pu, en effet, à la consultation de son dossier, se replonger dans l'atmosphère de la vie académique, retrouvant inscrit dans un rapport ces propos de couloir que l'on tient inconsidérément sous toutes les latitudes mais qu'aucune Stasi n'est là pour consigner... Faisant son miel de toutes ces rancoeurs et de tous ces ragots, l'officier sait aussi donner à l'occasion de judicieux conseils, encourager parfois à la modération comme s'il n'était là que pour veiller à la paix sociale dans l'institution... Ainsi, à l'agent " Montag ", historien et président de l'association des historiens de RDA qui veut empêcher la publication d'un ouvrage au prétexte qu'il parlerait trop d'« humanisme ", l'officier souffle: "Evitez d'être trop politique dans vos critiques, soyez plus souple». Et quand le même "Montag ", qui n'a pas su suivre ces conseils, se sent soudain bien seul parmi ses collègues, il lui reste son officier à qui confier sa solitude.

15 Le fréquent changement du personnel de la Stasi avait-il pour but d'éviter qu'une complicité ne s'établisse entre l'officier et son agent? C'est probable, et c'est la raison 
avancée pour que le lieutenant Rauch soit contraint de céder le dossier du Professeur Markov - un agent très spécial qui n'a jamais écrit aucun rapport et a refusé d'en écrire sur ses propres collègues, mais qui aimait à se moquer des "problématiques primitives " des historiens en bavardant avec son officier, lequel n'était autre qu'un ancien élève ${ }^{15}$.

Quoiqu'il en soit de l'authenticité des "aveux» de certains anciens agents de la Stasi (ces IM qui ont défrayé la chronique, comme « Donald », époux d'une grande dissidente, ou encore l'écrivain Sascha Anderson), selon lesquels leur relation avec leur officier respectif était fondée sur la confiance réciproque et un réel échange d'idées, ce type de rapport se vérifie plus d'une fois à l'examen attentif de dossiers. Que cela produise des personnalités "clivées ", tant du côté des agents que de leurs officiers, n'est pas une excuse (pour autant qu'il faille en trouver une), mais une explication non négligeable pour comprendre les divers aspects de la surveillance et ses potentialités.

L'instance morale

Il s'agit là probablement de la figure la plus inattendue, laquelle met en évidence une fonction éthique paradoxale puisque émanant d'une institution dont la finalité fait fi de toute morale. Cette fonction peut pourtant découler de celle de confident dont elle est le prolongement, ainsi que le montrent de façon irréfutable deux des dossiers que nous avons consultés. Tout en " pilotant » son agent (une étudiante "à la dérive ») à travers la vie nocturne de la bohème berlinoise qu'elle est chargée d'espionner, le major Erazim tente de la remettre sur le droit chemin. Le "profil psychologique " qu'il en dresse fait à l'évidence preuve de compassion et, cherchant à la stabiliser, il l'aide même à trouver un emploi (peut-être dans le but de la rendre " opérationnelle » dans un autre milieu, mais rien ne le laisse entendre). En ce sens, il oeuvrera en définitive contre les intérêts de la Stasi puisque, à peine "remise en selle ", l'étudiante refusera de continuer à rédiger des rapports, avouant se sentir mal à écrire sur des gens dont elle partage les idées...

Le dossier de l'agent « Rehbein » (que j'avais surnommé l'agent «sans qualités » dans mon étude précitée) est encore plus explicite et convaincant quant à une fonction occulte mais réelle de la Stasi.

20 L'agent « Rehbein » dirige l'Institut de philosophie de l'Académie des Sciences. Formé à cette discipline aux premiers jours de la RDA, il ne connaît guère que le diamat (matérialisme dialectique), mais a su très tôt compenser la faiblesse de son capital culturel en gagnant en capital politique ("Cette sous-catégorie du capital social qu'on peut appeler capital politique » selon Bourdieu $\left.{ }^{16}\right)$, c'est-à-dire en occupant des fonctions d'encadrement. Ayant rapidement compris le supplément de pouvoir qu'il pouvait tirer d'une collaboration avec la Stasi, il l'accepte sans difficulté et parle d'emblée abondamment. Mais en disant du mal systématiquement de tous ceux qui l'entourent, et en rapportant les pires ragots, cet informateur zélé excède les voeux de la Stasi et finit par perdre toute crédibilité aux yeux de son officier traitant. Ce dernier le qualifie très tôt de "carriériste rusé et sans scrupules [qui] exerce un pouvoir sans partage sur son institut ». Le dossier de «Rehbein » est émaillé de jugements négatifs sur lui et rédigés par son officier traitant qui consigne également ses propres tentatives pour "encadrer» le "philosophe", limiter son influence délétère sur l'Institut de philosophie où, note l'officier, "il n'est jamais question de politique, encore moins de philosophie ». De façon surprenante, car cela ne relève pas des attributions de la Stasi, l'incompétence du philosophe est soulignée et analysée à juste titre comme source de sa rivalité avec la presque totalité des membres de l'Institut, mieux formés que leur 
chef, ainsi que de ses ressentiments dont il rebat les oreilles de son officier traitant. Arrive le moment où le lieutenant Hoffmann perd tout espoir de rééduquer son agent ("Avec l'âge, ses défauts se sont accentués») et décide de mettre fin à sa collaboration. Licencié de la Stasi, «Rehbein" n'en continuera pas moins à exercer les fonctions de directeur de l'Institut de philosophie de l'Académie des sciences, et cela, malgré les avertissements de l'officier traitant ayant très clairement conseillé en haut lieu qu'on lui retire ses fonctions.

21 Dans ce cas, l'immoralisme de l'agent s'est finalement retourné contre la Stasi. Pis : intriguant par nature, «Rehbein » a fini par intriguer contre elle et obtenir victoire. Vraisemblablement protégé par le Parti, il conservera ses fonctions de directeur et, comme toujours en cas de désaccord, c'est la Stasi qui s'inclinera devant les décisions du Parti. Incontestablement, la lecture de ce dossier fait apparaître le lieutenant Hoffmann comme une figure morale et moralisatrice. Non seulement est-ce la seule personne qui glisse quelques vérités à « Rehbein » et met en doute ses attaques mais, comble du paradoxe, c'est lui qui intervient pour défendre les victimes du tout puissant patron de la philosophie est-allemande ${ }^{17}$ ! Instance morale, la Stasi apparaît ici également comme une instance d'arbitrage.

Ainsi, par delà la figure universelle du flic qu'il évoque, l'officier de la Stasi se révèle un produit archétypique du régime qui l'a engendré. Il en porte les marques et les stigmates: un conformiste faisant preuve de cette Anpassungsfähigkeit (capacité d'adaptation) qui n'est jamais tant décriée que lorsqu'elle n'a plus lieu d'être, un citoyen dépourvu d'esprit critique ou de faculté de penser par soi-même, respectueux de l'ordre établi et donc du pouvoir, prêt à tout pour maintenir l'ordre imposé par ce dernier au nom de cette fin qui justifie tous les moyens; mais aussi un témoin souvent indigné de « la face cachée » des hommes. Dans l'un des rares témoignages d'anciens de la Stasi, un officier évoque le cas d'un de ses agents, un chef d'entreprise, qu'il avait démasqué comme agent double pour le compte du BND (service de renseignement ouest-allemand). De cet homme qu'il nomme « S., le parasite », il dit : «Il ne s'agissait pas ici d'une personne qui, par l'agitation ou la calomnie, avait violé le code pénal de la RDA, mais d'un parasite (...). A mes yeux, c'était un bonhomme qui avait besoin d'argent pour entretenir ses maîtresses et qui avait su se monter suffisamment en épingle. (...) S. a été échangé [contre des Deutschmarks ouest-allemands $\mathrm{NdR}$ ] avant la date prévue. Je n'ai jamais compris si c'était effectivement un informateur de pointe, un agent du BND ou simplement un parasite $d u$ socialisme. On le saura un jour. S. pourrait se trouver devant moi et se faire passer pour un révolutionnaire, ou pour un militant anti-communiste. Et les gens le croiront. Alors que son seul souci, c'étaient les femmes et l'argent $»^{18}$. Constat lucide et empreint de réprobation morale de la part d'un homme qui, deux pages auparavant, avouait: "Il fallait protéger les accusés, souvent des adversaires impénitents du socialisme, contre celui qui menait l'interrogatoire lorsqu'il s'agissait de quelqu'un de jeune et d'inexpérimenté. Toute l'audition et tout l'interrogatoire devaient être enregistrés sur bande magnétique. (...) Le magnétophone était un moyen de pression sur les interrogateurs qui avaient la main trop leste. Il a fait ses preuves. Il a permis de limiter les actes de violence ou les mauvais traitements $»^{19}$.

Oscillant entre la posture de chien de garde du régime et celle de personnage moral (aux pratiques immorales), l'officier de la Stasi est à l'image de l'Etat qu'il sert: une figure simple dans l'exercice de ses fonctions répressives, complexe dans la mise en oeuvre contradictoire de principes puisant aux origines fondatrices du régime. Ce sont précisément ces contradictions qui permettent de comprendre ses succès en matière de 
recrutement d'informateurs et de quadrillage de la société. A son autorité policière fondée sur sa fonction répressive et visant à prévenir et neutraliser l'opposition, la Stasi avait ajouté une autorité de type plus idéologique. Elle était parvenue à faire collaborer une fraction décisive de la société en s'appuyant sur la légitimité qu'ont tous les services de renseignements dont la fonction est d'assurer la sécurité du pays. De ce point de vue, l'argument de la loyauté (qui renvoie aussi bien à l'adhésion idéologique qu'au rapport à la RFA, l'Allemagne rivale, ou au civisme par conformisme) avancé tant par des informateurs de la Stasi que par des officiers eux-mêmes à la fin du régime, correspondait bien à une réalité. Sans entrer dans un débat qui nous entraînerait audelà de l'objectif de cette contribution, force est d'admettre que, contrairement à l'Union soviétique, la RDA ne s'est pas effondrée de l'intérieur, mais sous la pression de facteurs exogènes. Et cela, bien que sa chute ait été spectaculaire et foudroyante, et bien que tous les symptômes annonciateurs de décomposition aient été présents. C'est là la meilleure preuve du phénomène d'intériorisation des contraintes, né de cette surveillance dont l'officier traitant a été l'artisan, et qui a participé du mode de gouvernement d'un régime jamais menacé par une véritable opposition.

\section{NOTES}

1. . On relèvera à ce sujet la fonction, là encore unique, de l'institution garante de la conservation et de la communication de ces archives, branche des archives nationales (Bundesarchiv), généralement appelée suivant le nom de son responsable (Gauckbehörde, puis Birthlerbehörde), qui a aussi pour mission d'abriter des historiens et de favoriser leurs travaux.

2. . Gellately R., " Denunciations in Twentieth-Century Germany : Aspects of Self Policing in the Third Reich and the German Democratic Republic », in Journal of Modern History, n68, décembre 1996.

3. . Hrabal B., Peurs totales, Critérion, 1991.

4. . Citons au moins deux sources, dont l'une existe en français : récit recueilli par Hahn R.O., Au nom du peuple. Un officier de la Stasi raconte, Albin Michel, collection « Histoire à deux voix », 1999 (traduit par Olivier Mannoni) et le documentaire filmique « Der Schwarze Kasten. Psychogramm eines Taters », de J. Feindt et T. Trempe, 1992.

5. . Combe S., Une société sous surveillance. Les intellectuels et la Stasi, Paris, Albin Michel, $1999,263 \mathrm{p}$.

6. . Une telle étude ne manquerait pas d'intérêt, mais serait-elle possible ? Le mode de classement des archives de la Stasi ne s'y prête guère. Comme toutes les archives policières, elles sont classées par noms de personnes et non par noms de collectivités. 7. . La répression des intellectuels communistes (c'est-à-dire non dissidents mais critiques) commence ouvertement avec l'arrestation, en 1956, du jeune philosophe Wolfgang Harich et se poursuit avec la disgrâce du philosophe Ernst Bloch et de son entourage, à l'université de Leipzig, qui ne prendra fin qu'après plusieurs arrestations et la « normalisation » du milieu universitaire (1958). Ernst Bloch passera à l'Ouest à l'été 1961, juste avant la construction du mur de Berlin. 
8. . Ces Inoffizielle Mitarbeiter, généralement appelés par les initiales I.M., correspondaient à près de 200000 personnes à la chute du régime. On estime qu'environ un citoyen est-allemand sur 3 a été, à un moment où à un autre, collaborateur de la Stasi. Mais tous les citoyens de RDA ne l'ont pas été de manière identique. Comme ce n'est pas là l'objet de cet article, je me permets de renvoyer à mon étude déjà citée (Une société sous surveillance...) qui examine précisément les différents cas de figures.

9. . On a beaucoup parlé des quelques cas recensés où l'un des époux aurait surveillé l'autre. S'ils existèrent bel et bien, ils furent néanmoins rares mais ils frappèrent d'autant plus les esprits que c'est ce pouvoir d'abolition des frontières du privé/public des polices secrètes qui accentue le sentiment de vulnérabilité de l'individu, privé de tout refuge et de toute intimité.

10. Foucault, M. Surveiller et punir, Gallimard, 1975

11.. Par source, il faut entendre les rapports des informateurs non officiels, qu'il s'agisse de rapports écrits ou oraux, dont l'officier de la Stasi va faire la synthèse.

12. . Stephen, A, Im Visier der FBI. Deutsche Exilschriftsteller in den Akten amerikanischer Geheimdienste, J.B. Metzler, 1995.

13. . Equivalent en France du CNRS.

14. . Allusion à l'ouvrage de Engels sur Les origines de la famille.

15. . Historien réputé et antifasciste persécuté par la Gestapo, Walter Markov avait fait partie de ces intellectuels « progressistes » que Hans Georg Gadamer, premier recteur de l'université de Leipzig après guerre, avait recrutés.

16. . Voir l'intervention de Pierre Bourdieu en octobre 1989 à l'université Humboldt dans Die Intellektuellen und die Macht, VSA, 1991.

17. . Relevons le comique de l'affaire : «Rehbein » s'était fait de nombreux amis parmi les philosophes occidentaux auprès desquels il passait pour un intellectuel communiste critique et ouvert. Non seulement était-il pratiquement le seul à pouvoir se rendre à l'étranger (il s'opposait systématiquement à ce qu'un autre que lui-même s'y rende), mais, en tant qu'agent de la Stasi, il ne redoutait pas cette dernière et pouvait se permettre de jouer à l'extérieur le quasi-dissident. Ce rôle-là masqua probablement sa totale incompétence scientifique.

18. . Hahn R.O., Au nom du peuple, op. cit. p.75.

19. . Ibid., p.69.

INDEX

Index géographique : Allemagne

Mots-clés : police, Renseignement, surveillance

Index chronologique : guerre froide 